\title{
Menstrual and reproductive outcomes after use of balloon tamponade for severe postpartum hemorrhage
}

\author{
Choi Wah KONG ${ }^{*}$ iD and William Wing Kee TO
}

\begin{abstract}
Background: The use of intrauterine balloon tamponade to manage postpartum hemorrhage is increasing. However, there is lack of studies on the menstrual and reproductive outcomes after such treatment. The purpose of this study is to explore the menstrual and reproductive outcomes for patients who had been managed by intrauterine balloon tamponade for severe postpartum hemorrhage in her index pregnancy.

Methods: All patients who had delivered in United Christian Hospital from January 2011 to June 2016 with severe postpartum hemorrhage $(\mathrm{PPH}$ ) (blood loss $>=1 \mathrm{~L}$ ) were identified by the labour ward delivery registry and a comprehensive obstetric database. Patients who had intrauterine balloon tamponade inserted were compared with those managed solely by uterotonic agents as controls. Patients who had hysterectomy or additional procedures performed, such as compression sutures or uterine artery embolization were excluded from both groups. A questionnaire on menses, fertility and reproductive outcomes was mailed to both groups of patients. Those that had not replied within 4 weeks would receive a telephone survey.
\end{abstract}

Results: A total of 39 patients in the balloon tamponade group and 161 patients in the control group were recruited, which represented $87.0 \%$ of all eligible patients within the study period. The median follow up period was 45 months. All patients in the balloon tamponade group had return of menses after delivery. The majority of the patients (87.2\%) in the balloon tamponade group had normal menstrual patterns in the 12 months after the index delivery as well as in the most recent 12 months. After excluding the patients with contraception, the subsequent pregnancy rate was $42.9 \%$ $(9 / 21)$ in the balloon tamponade group compared to $45.9 \%(28 / 61)$ in the control group $(p=0.81)$. Among the 9 subsequent pregnancies in the balloon tamponade group, there were two miscarriages, one scar pregnancy, one induced abortion, while the remaining five were normal pregnancies with full term deliveries without intrauterine growth restriction. The majority of patients replied that they were satisfied with using Bakri balloon for PPH management in their index pregnancy.

Conclusions: Intrauterine balloon tamponade for the management of severe PPH appeared to pose little adverse effects on subsequent menstrual and reproductive function.

Keywords: Intrauterine balloon tamponade, Fertility, Menstruation, Postpartum hemorrhage, Pregnancy

\footnotetext{
* Correspondence: melizakong@gmail.com

Department of Obstetrics and Gynaecology, United Christian Hospital, 130

Hip Wo Street, Kwun Tong, Hong Kong
}

(c) The Author(s). 2018 Open Access This article is distributed under the terms of the Creative Commons Attribution 4.0 International License (http://creativecommons.org/licenses/by/4.0/), which permits unrestricted use, distribution, and reproduction in any medium, provided you give appropriate credit to the original author(s) and the source, provide a link to the Creative Commons license, and indicate if changes were made. The Creative Commons Public Domain Dedication waiver (http://creativecommons.org/publicdomain/zero/1.0/) applies to the data made available in this article, unless otherwise stated. 


\section{Background}

The incidence of postpartum hemorrhage is around 5\% and is continuously increasing worldwide due to increase in patients with advanced maternal age, multiple pregnancies, caesarean deliveries and previous caesarean sections with abnormal placentation [1-4]. Using intrauterine balloon to manage severe postpartum hemorrhage $(\mathrm{PPH})$ is shown to be effective and can decrease the rate of hysterectomy [58]. As a result, intrauterine balloon has been more and more commonly incorporated into severe PPH protocols. It has become an integral part of the "HEMOSTASIS" management algorithm advocated in the United Kingdom [9]. However, follow-up studies on the menstrual and reproductive outcomes after the use of balloon tamponade are minimal. There are only two studies in the literature that has specifically reviewed patients' menses after the use of balloon tamponade, of which one was a case series that included only 5 patients [10] while the other included 33 women but was only published as electronic poster [11]. For the reproductive outcomes, there are only five studies available that have reported a total of 14 patients' subsequent pregnancy and delivery after the use of balloon tamponade [11-14]. However, the details on subsequent pregnancy outcome such as the gestational age at delivery were not reported in at least half of the cases. Therefore this study aims at reviewing an unselected cohort of patients who had been managed with intrauterine balloon for severe $\mathrm{PPH}$ in their index pregnancy over a period of 66 months. Using this larger sample size, we attempted to evaluate the details of the patients' menstrual functions, fertility and reproductive outcomes, in order to verify the long term impact of balloon tamponade on these patients with severe postpartum hemorrhage in their previous pregnancies.

\section{Methods}

This was a retrospective cohort study. All pregnant patients with deliveries in United Christian Hospital from January 2011 to June 2016 who had severe PPH (blood loss $>=1 \mathrm{~L}$ ) were recruited. These patients were identified from our previous studies $[15,16]$ and their demographic and clinical data were reviewed. Patients who had uterine balloon inserted were compared with those severe PPH patients who were managed solely by uterotonic agents which served as the control group.

The department protocol of management of severe postpartum hemorrhage was attached in Additional file 1. The Bakri intrauterine balloon catheter (Cook Medical, Bloomington [IN], US) was the only available balloon tamponade for this purpose in our department. The procedure for application of the Bakri balloon was in accordance with that generally described in the literature [5-7]. When the balloon was inserted during a caesarean section, the distal end of the balloon shaft was passed through the cervical opening with an assistant pulling the end per vagina. The amount of saline instilled was the amount considered sufficient by the operator to produce a "positive tamponade test" [5, 9], up to maximum of $500 \mathrm{ml}$. If bleeding was arrested after balloon inflation, the balloon tamponade was removed within $24 \mathrm{~h}$ after insertion.

For patients who had intrauterine balloon tamponade attempted or inserted, if they had hysterectomies or additional procedures performed such as compression sutures and uterine artery embolization (UAE), they would be excluded in both the balloon tamponade group and the control group as these additional procedures themselves may affect the menstrual and reproductive outcomes.

A questionnaire on menstrual patterns, fertility and reproductive outcomes was mailed to both groups of patients (see Additional files 2 and 3). A consent form was attached to the questionnaire and written consent was obtained. The questionnaire and consent form were designed in two languages: traditional Chinese and English. They were mailed to the patient with language appropriate to her need. Patients were asked to return the completed questionnaire and consent form by mail with a pre-paid return envelope or by fax to a specific line to the principal investigator. If no reply was received from the patient after 4 weeks, the principal investigator would attempt to contact the patient by phone to conduct a telephone survey and the same questions as in the mailed questionnaire would be asked. Patients would also be contacted by phone by the principal investigator to clarify any queries in their questionnaire reply if deemed necessary. Additional data was collected from the patients involved in the previous studies, for the current study. Ethics approval for this study was granted by the Kowloon Central/ Kowloon East Ethics Committee Board of the Hospital Authority, Hong Kong. (KC/ KE-16-0169/ER-1).

The primary outcome was the percentage of patients that had normal menses after delivery while the secondary outcomes were the percentage of patients that had subsequent pregnancies. SPSS for Windows package (SSPS Inc., Chicago, IL Version 23.0) was used for data entry and analysis. The differences between continuous variables were analyzed using student's t-test. The differences between discrete variables were analyzed by Chi-square test or Fisher's exact test when appropriate. A $p$-value of $<0.05$ was considered statistically significant.

\section{Results}

There were a total of 25,343 deliveries during the study period. The frequency of primary $\mathrm{PPH}$ with an estimated blood loss exceeding $500 \mathrm{~mL}$ was $6.15 \%$ ( $n$ $=1558)$. The frequency of massive PPH with an estimated blood loss exceeding $1 \mathrm{~L}$ was $1.09 \%(n=277)$. 
Among these patients, Bakri balloon tamponade was attempted in 60 (21.7\%) of them, among which, there was 9 cases with peripartum hysterectomy performed and in which one case had maternal death. Balloon tamponade was successful in arresting PPH without hysterectomy in 51 out of 60 patients (85\%). In these 51 patients with balloon tamponade inserted, 6 patients required additional procedures including $\operatorname{UAE}(n=2)$, compression sutures $(n=2)$ and both $(\mathrm{n}=2)$. A total of 185 patients had severe $\mathrm{PPH}$ that were successfully managed solely with uterotonics served as the control group (Fig. 1).

Among the 45 patients in the balloon tamponade group, 39 completed the questionnaire survey giving a response rate of $86.7 \%$. Within the control group, 161 patients completed the questionnaire survey giving a comparable response rate of $87.0 \%$. The overall recruitment rate was 200/230 (87.0\%), of which 141 patients replied to the written questionnaire and 59 patients replied to the telephone survey. None of the patients that could be contacted refused to participate in the survey. There were no significant differences in the basic epidemiological characteristics or clinical history between the recruited subjects and those patients that could not be contacted.

The mean interval between the survey and the index pregnancy was 47.7 (SD 16.5) months for the balloon tamponade group and 44.1 (SD 17.0) months for the control group. There were no significant differences in basic epidemiological parameters including the maternal age, parity, mode of delivery, or the aetiology of the severe $\mathrm{PPH}$ between the two groups of patients. The balloon tamponade group had significantly more blood loss and higher incidence of blood product transfusion than the control group. There was no difference in the incidence of postpartum endometritis between the two groups (Table 1).

One patient in the control group was diagnosed to have polycystic ovarian syndrome with prolonged periods of amenorrhoea prior to her index pregnancy, which was induced by in-vitro fertilization. She reported secondary amenorrhoea for over 4 years up to the time of the survey, though she has been breast feeding for over 20 months in the postpartum period. Her prolonged amenorrhoea was ascribed to her chronic anovulation similar to her pre-pregnancy state. When the menstrual patterns were compared between the two groups, it could be seen that after excluding the patient with permanent amenorrhoea, the menstrual pattern of both groups of patients were largely normal in the 12 months after the index delivery as well as in the most recent 12 months. (Tables 2 and 3).

Concerning the reproductive outcomes, 46.2\% (18/ 39) of the patients in the balloon tamponade group practised contraception after the index pregnancy compared with $62.1 \%(100 / 161)$ of the patients in the control group. After excluding those with contraception, nine patients $(42.9 \%)$ in the balloon tamponade group had subsequent pregnancies, of

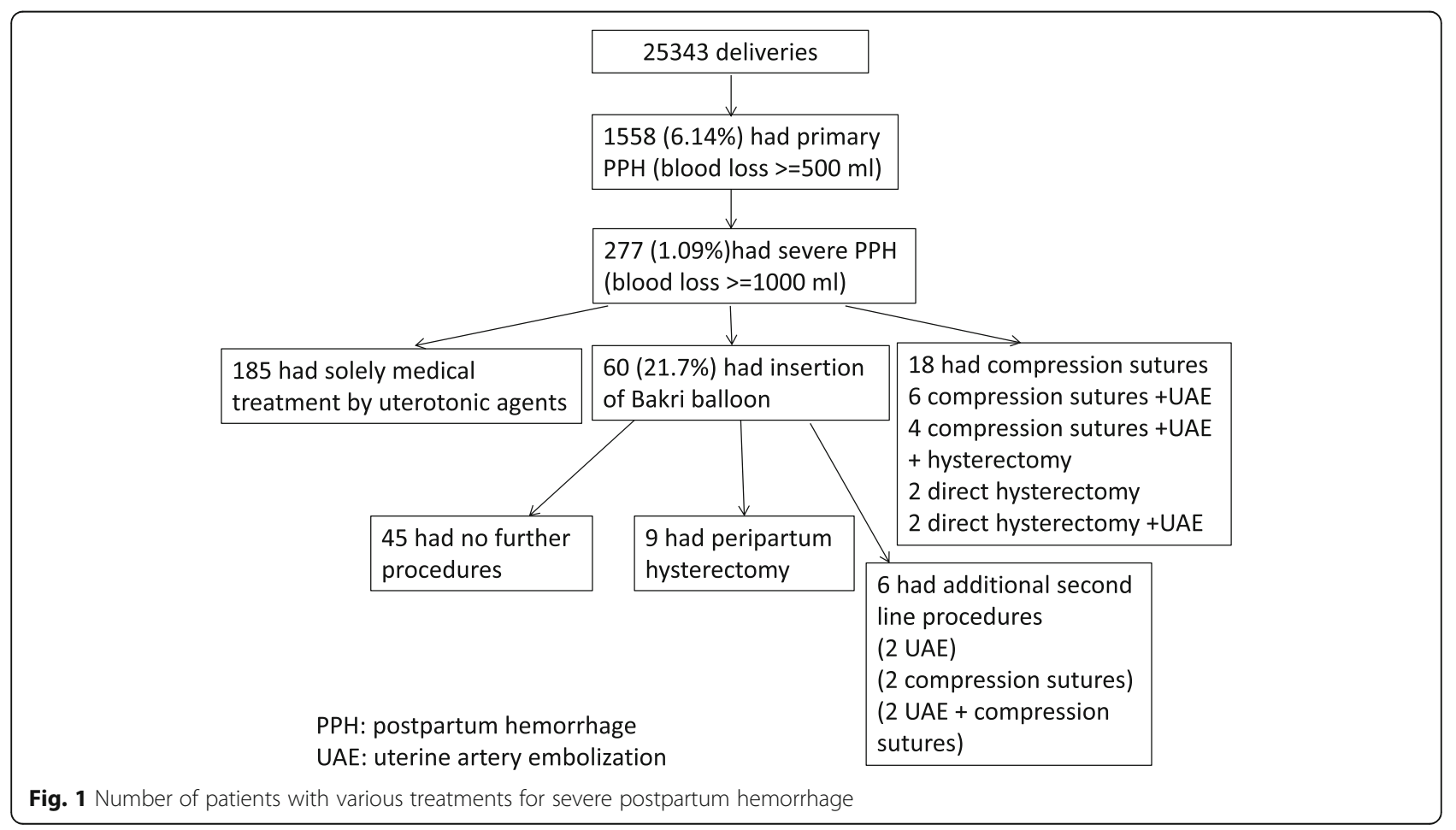


Table 1 Clinical parameters between Bakri balloon group vs control group

\begin{tabular}{|c|c|c|c|}
\hline & Bakri balloon only $(n=39)$ & Control $(n=161)$ & $p$-value; MD; $(95 \% \mathrm{Cl})$ \\
\hline Maternal age (years) & $33.9(4.00)$ & $34.6(4.98)$ & $0.442 ;-0.66(-2.35$ to 1.03$)$ \\
\hline Gestation at delivery (weeks) & $38.1(2.07)$ & $38.3(2.43)$ & $0.78 ;-0.12(-0.95$ to 0.71$)$ \\
\hline \multicolumn{4}{|l|}{ Parity } \\
\hline Primiparous & $19(48.7 \%)$ & $71(44.1 \%)$ & \multirow[t]{2}{*}{0.60} \\
\hline Multiparous & $20(51.3 \%)$ & $90(55.9 \%)$ & \\
\hline Multiple pregnancy & $7(17.9 \%)$ & $17(10.6 \%)$ & 0.27 \\
\hline Previous caesarean section & $5(12.8 \%)$ & $52(32.3 \%)$ & ${ }^{*} 0.02$ \\
\hline \multicolumn{4}{|l|}{ Mode of delivery } \\
\hline Normal vaginal & $9(23.1 \%)$ & $27(16.8 \%)$ & \multirow[t]{3}{*}{0.56} \\
\hline Instrumental & $1(2.6 \%)$ & $8(5.0 \%)$ & \\
\hline Caesarean section & $29(74.3 \%)$ & $126(78.3 \%)$ & \\
\hline \multicolumn{4}{|l|}{ Cause of severe PPH } \\
\hline Uterine atony & $24(61.5 \%)$ & $115(71.4 \%)$ & \multirow[t]{3}{*}{0.38} \\
\hline Placenta praevia/accreta & $12(30.8 \%)$ & $33(20.5 \%)$ & \\
\hline Genital tract trauma & $3(7.7 \%)$ & $13(8.1 \%)$ & \\
\hline Total blood loss (ml) & $1812(748)$ & $1440(413)$ & ${ }^{*}<0.001 ; 371$ (197 to 545) \\
\hline Blood product transfusion & 35 (89.7\%) & 91 (56.5\%) & ${ }^{*}<0.001$ \\
\hline Coagulopathy & $11(28.2 \%)$ & $34(21.1 \%)$ & 0.34 \\
\hline Presence of endometritis after delivery & $1(2.6 \%)$ & $3(1.9 \%)$ & 0.583 \\
\hline
\end{tabular}

MD Mean difference, $\mathrm{Cl}$ Confidence interval

*statistically significant

which 5 were live births, 2 were miscarriages, 1 was a scar ectopic pregnancy and 1 had termination of pregnancy due to social reasons. For the 5 livebirths, all were term live births without intrauterine growth restriction and 2 patients had $\mathrm{PPH}$ again in their subsequent pregnancies but both were managed successfully with oxytocin. Within the control group, 28 patients (45.9\%) had subsequent pregnancies, of which 16 were live births, 5 were miscarriages, 2 had ectopic pregnancies with one of them being a scar ectopic, and 5 had termination of pregnancy. For the 16 livebirths, 2 were born preterm at 31 and 36 week of gestation and 6 patients had PPH again in their subsequent pregnancies which were managed by oxytocin. There were no significant differences between the subsequent pregnancy rate and live birth rate between the Bakri balloon and control group ( $p=0.81$ and $p=0.83)$. (Table 4$)$.

The maternal satisfaction rate with the use of balloon tamponade was 37/39 (94.9\%). Only 2 patients expressed dissatisfaction with the balloon tamponade treatment in the survey. One of them had secondary $\mathrm{PPH}$ requiring surgical evacuation yielding decidualized tissue compatible with postpartum endometritis. Despite a full course of antibiotic treatment, the patient continued to have prolonged lochia which lasted for up to 3 months post-delivery. In the other patient, she was dissatisfied with Bakri balloon treatment due to pain experienced during the insertion of the balloon.

\section{Discussion}

The current survey of patients with Bakri balloon insertion for severe $\mathrm{PPH}$ in their index pregnancies confirmed that the majority of them would have normal menstrual patterns and reproductive performance.

$\mathrm{PPH}$ continues to be one of the world's leading causes of maternal mortality [17]. Traditionally, peripartum hysterectomy would be performed in patients with massive PPH as a life-saving rescue procedure in those who failed to respond to uterotonics. However, due to the high associated morbidity associated with peripartum hysterectomy, various conservative surgical procedures have been developed to reduce the need for hysterectomy, including external compression sutures, selective devascularization by surgical ligation or radiological embolization of the uterine and pelvic arteries [5, 18-20]. However, most of these studies focused on the success rates in avoiding hysterectomy, with little direct data available on the actual preservation of fertility in these women, including the return of menses, the pregnancy rate, or the outcome of subsequent pregnancies. In addition, further limitations to gathering such data could result from the possible bias arising from the 
Table 2 Menstrual pattern within 12 months postpartum between Bakri balloon group vs control group

\begin{tabular}{|c|c|c|c|}
\hline & Bakri balloon only $(n=39)$ & Control $(n=161)$ & p-value; MD; $(95 \%$ Cl) \\
\hline $\begin{array}{l}\text { Duration of breastfeeding postpartum } \\
\text { (months) }\end{array}$ & 4.41 (6.05); median 1.5 range $0-24 \mathrm{~m}$ & 3.25 (4.44); median 2.0 range $0-30 \mathrm{~m}$ & $0.176 ; 1.16(-0.52$ to 2.84$)$ \\
\hline Return of menses after delivery (months) & $4.43(4.68)$ & $4.95(3.24)$ & $0.42 ;-0.51(-1.77$ to 0.74$)$ \\
\hline \multicolumn{4}{|l|}{$\begin{array}{l}\text { Menstrual pattern within } 12 \text { months } \\
\text { postpartum }\end{array}$} \\
\hline No return of menses & 0 & $1^{\dagger}$ & 1.00 \\
\hline Cycle regularity & & & 0.47 \\
\hline Regular $28( \pm 7)$ day cycles & $34(87.2 \%)$ & $141(88.1 \%)$ & \\
\hline $\begin{array}{l}\text { Short (<21 day) or long ( }>35 \text { day) } \\
\text { cycles }\end{array}$ & $3(7.7 \%)$ & $16(10.0 \%)$ & \\
\hline Totally irregular cycles & $2(5.1 \%)$ & $3(1.9 \%)$ & \\
\hline Duration of flow & & & 0.41 \\
\hline Little flow $<2$ days & $0(0.0 \%)$ & $3(1.9 \%)$ & \\
\hline Normal flow (3-7 days) & $35(89.7 \%)$ & $148(92.5 \%)$ & \\
\hline Prolonged flow (> 7 days) & $4(10.3 \%)$ & $9(5.6 \%)$ & \\
\hline Amount of flow & & & 0.172 \\
\hline Heavy & $6(15.4 \%)$ & $23(14.4 \%)$ & \\
\hline Normal & $26(66.7 \%)$ & $124(77.5 \%)$ & \\
\hline Scanty & $7(17.9 \%)$ & $13(8.1 \%)$ & \\
\hline Presence of dysmenorrhoea & & & 0.46 \\
\hline No dysmenorrhoea & $31(79.5 \%)$ & $136(85.0 \%)$ & \\
\hline Mild & $8(20.5 \%)$ & $22(13.8 \%)$ & \\
\hline Severe requiring medication & $0(0.0 \%)$ & $2(1.2 \%)$ & \\
\hline
\end{tabular}

MD Mean difference, $\mathrm{Cl}$ Confidence interval

tOne patient with prolong secondary amenorrhoea was excluded from subsequent analysis

reluctance of PPH patients to pursue a subsequent pregnancy [21].

Of the 21 successful pregnancies in our cohort, 8 were reported to have PPH controlled by oxytoxics. From a large North American cohort, it was shown that women with a history of PPH had a 3-fold increased risk of PPH in their second pregnancy (15\%) compared with unaffected women $(5.0 \%)$; and in a third pregnancy, the risk rose to $26.6 \%$ after 2 previously affected pregnancies. In addition, it was observed that recurrent PPH in subsequent pregnancies was particularly higher in those with a previous severe $\mathrm{PPH}$. Such observations were compatible with what was observed in our survey [22]. In a case series that studied pregnancy outcome in women who had radiological pelvic arterial embolization for postpartum hemorrhage, it was also shown that there was a significantly higher rate of placenta accreta as compared to women without embolization in their previous pregnancy $(23.5 \%$ vs $0 \%)$. This high incidence of recurrent PPH was probably more related to predisposing risk factors that had existed even for the index pregnancy [23]. Thus, regardless of the technique used in the management of PPH, the risk of recurrent PPH in subsequent pregnancies is substantial [21].
So far, there were more studies in the literature targeted at reviewing menstrual and fertility outcomes after pelvic vascular ligation, radiological embolization and compression sutures for PPH than after balloon tamponade. For instance, a relatively large cohort of 68 patients with hypogastric artery ligation for $\mathrm{PPH}$ reported no increase in subsequent subfertility when compared to controls [24], and another series of 53 women who had uterine artery embolization also showed no statistically significant differences of occurrence of pregnancy between the embolized and nonembolized groups [25]. Uterine compression sutures have been shown to be associated with subsequent uterine synechiae formation as demonstrated by hysteroscopy in up to $26 \%$ of the cases, though the impact of this on pregnancy outcome is uncertain [26]. However, such synechiae formation has not been reported to be associated with balloon tamponade so far. A recent review has raised the possible increased risks of endometritis in those with balloon management [27]. In our cohort, all the Bakri balloons were removed within $24 \mathrm{~h}$ after insertion and there was no significantly increase in this incidence after Bakri balloon insertion. Therefore, Bakri balloon appears to be 
Table 3 Menstrual pattern in recent 12 months postpartum between Bakri balloon group vs control group

\begin{tabular}{|c|c|c|c|}
\hline & Bakri balloon only $(n=39)$ & Control $(n=161)$ & p-value; MD (95\% Cl) \\
\hline Mean interval from delivery to survey (months) (SD) & $47.7(16.5)$ & $44.1(17.0)$ & $0.26 ; 3.44(-2.52$ to 9.40$)$ \\
\hline Cycle regularity & & & 0.20 \\
\hline No menses & 0 & $1^{+}$ & \\
\hline Regular $28( \pm 7)$ day cycles & $34(87.2 \%)$ & $127(79.4 \%)$ & \\
\hline Short ( $<21$ day) or long ( $>35$ day) cycles & $2(5.1 \%)$ & $25(15.6 \%)$ & \\
\hline Totally irregular cycles & $3(7.7 \%)$ & $8(5.0 \%)$ & \\
\hline Duration of flow & & & 0.95 \\
\hline Little flow $<2$ days & $0(0.0 \%)$ & $6(3.7 \%)$ & \\
\hline Normal flow (3-7 days) & $34(87.2 \%)$ & $143(89.4 \%)$ & \\
\hline Prolonged flow (> 7 days) & $5(12.8 \%)$ & $11(6.9 \%)$ & \\
\hline Amount of flow & & & 0.46 \\
\hline Heavy & $6(15.4 \%)$ & $28(17.5 \%)$ & \\
\hline Normal & $27(69.2 \%)$ & $118(73.8 \%)$ & \\
\hline Scanty & $6(15.4 \%)$ & $14(8.7 \%)$ & \\
\hline Presence of dysmenorrhoea & & & 0.36 \\
\hline No dysmenorrhoea & $31(79.5 \%)$ & $130(81.2 \%)$ & \\
\hline Mild & $8(20.5 \%)$ & $24(15.0 \%)$ & \\
\hline Severe requiring medication & $0(0.0 \%)$ & $6(3.8 \%)$ & \\
\hline
\end{tabular}

MD Mean difference, $\mathrm{Cl}$ Confidence interval, SD Standard deviation

tOne patient with prolong secondary amenorhoea was excluded from subsequent analysis

safe and has no long term effect on subsequent menstrual and reproductive function. A systematic review on the fertility rates and subsequent pregnancy outcome after conservative surgical procedures for $\mathrm{PPH}$ has reported that compressive sutures were associated with good pregnancy outcomes but an increasing rate of subsequent repeat caesarean section, while pelvic artery embolization is more likely to be associated with subsequent placental disorders, leading to fetal growth restriction, preeclampsia, and placenta accreta and/or praevia [21].
There were only two studies that had assessed the menstrual function after balloon tamponade. In a small series of five patients who underwent balloon tamponade procedure for severe $\mathrm{PPH}$, all of them had return of menses within 8 week after delivery or after cessation of breast feeding, with normal amount and duration of menses [10]. Another larger cohort of 33 women reported $81.8 \%$ had regular menses after balloon tamponade insertion, though this study was only published as electronic poster [11]. There were also only scanty reports of pregnancy outcome after balloon tamponade

Table 4 Reproductive outcomes between Bakri balloon group vs control group

\begin{tabular}{llll}
\hline & Bakri balloon only $(\mathrm{n}=39)(\mathrm{SD})$ & Control $(\mathrm{n}=161)(\mathrm{SD})$ & $\mathrm{p}$-value; MD; $95 \% \mathrm{Cl}$ \\
\hline Use of contraception & $18(46.2 \%)$ & $100(62.1 \%)$ & 0.07 \\
Subsequent pregnancies\# & $9(42.9 \%)$ & $28(45.9 \%)$ & 0.81 \\
Occurrence after index deliveryf(months) & $28.4(13.0)$; range 11-48 & $28.6(10.9) ;$ range $10-52$ & $0.98 ;-0.13 ;-8.99$ to 8.74 \\
$\quad$ Miscarriage & $2(9.5 \%)$ & $5(8.2 \%)$ & 1.00 \\
$\quad$ Termination & $1(4.8 \%)$ & $5(8.2 \%)$ & 1.00 \\
Ectopic & $1(4.8 \%)$ & $2(3.3 \%)$ & 1.00 \\
Deliveries & $5(23.8 \%)$ & $16(26.2 \%)$ & 0.83 \\
$\quad$ Vaginal delivery & 4 & 11 & 6 \\
$\quad$ Caesarean section & 1 & 6 & \\
$\quad$ Presence of PPH & 2 & & \\
\hline
\end{tabular}


insertion. The pregnancy outcome of the above small series of five patients reported successful term pregnancies in all of them, though one of them had recurrent PPH controlled by oxytoxics [10]. The authors concluded that the uterine-specific Bakri balloon should pose minimal effect on menses, fertility and future pregnancies. In another French series of 49 women who had intrauterine balloon tamponade with an overall $65 \%$ success rate, it was reported that two women had a subsequent full-term pregnancy without recurrence of postpartum hemorrhage [14]. In another report of 2 cases using the Roush balloon, one woman was reported to have a subsequent uncomplicated pregnancy but the details were not given [12]. Moreover, among 5 patients who underwent uterine sandwich technique with compression sutures followed by Bakri balloon insertion for uterine atony, it was reported that 2 of the women had subsequent successful pregnancies, though one of them had recurrent PPH due to uterine atony [13]. In the cohort of 33 women as mentioned above, it was reported that 4 patients $(19.0 \%)$ with subsequent pregnancy and all the deliveries were uncomplicated [11]. Although not all the clinical details of the subsequent pregnancies were available in all of the above studies, their reports supported our findings that the use of intrauterine balloon tamponade did not affect subsequent menstrual and reproductive outcomes.

For other complications of Balloon tamponade, Franklin-Dumont et al. compared 13 patients with Bakri balloon inserted for postpartum hemorrhage with 351 patients without Bakri balloon inserted and found significantly increased in rate of endometritis after the use of Bakri balloon [27]. In our cohort, all the Bakri balloons were removed within $24 \mathrm{~h}$ after insertion and there was no significantly increase in the incidence of endometritis after Bakri balloon insertion. Therefore, Bakri balloon appears to be safe and has no long term effect on subsequent menstrual and reproductive function.

The strength of our current study was its focus on reviewing specifically the menstrual pattern and subsequent pregnancy outcome of this target group, and our data have comprehensively described the reproductive performance after specifically excluding involuntary subfertility in this cohort, instead of merely reporting the number of pregnancies as they occurred. Apparently, our cohort had reported the largest number of subsequent pregnancies reported so far in the literature. The median follow up time was around 45 months, which should be adequate to demonstrate any long term impact of the previous balloon procedure on menstrual or reproductive function.

The limitations of this study included the risk of selection bias in this cohort as the characteristic of the group of women with Bakri balloon was somewhat different from the control group, with higher blood loss and a higher incidence of blood product transfusions. However, while the higher blood loss was obviously the indication for balloon tamponade management in the first place, even with this potential confounding factor, the women in the balloon group did not have poorer menstrual and fertility outcome compared with the control group, implying that intrauterine balloon management should pose no effects on these outcomes. Another potential weakness of this cohort was the inability to assess the natural timing for return of menses after the procedure, as in many of the women, the return of menses was delayed due to breast feeding. There may also be recall bias for the menstrual outcome as the mean time interval from delivery to the survey was close to 4 years but the recall bias for subsequent pregnancy outcome should be minimal. Finally, a larger cohort would be needed to evaluate the risks of recurrent $\mathrm{PPH}$ or other obstetric complications in subsequent pregnancies.

\section{Conclusions}

Intrauterine balloon tamponade for the management of severe PPH appeared to pose little adverse effects on subsequent menstrual and reproductive function. The pregnancy outcome was similar to those managed conservatively with uterotonic agents alone. Intrauterine balloon tamponade should be recommended to be used as second line treatment for severe postpartum hemorrhage.

\section{Additional files}

Additional file 1: Department protocol for severe PPH. This file is the department protocol for severe postpartum hemorrhage in United Christian Hospital. (DOCX 21 kb)

Additional file 2: Questionnaire on menstrual patterns, fertility and reproductive outcomes after severe postpartum hemorrhage (balloon group). This file is the questionnaire that mailed to the patients with evere postpartum hemorrhage that were managed by balloon tamponade. (DOCX $30 \mathrm{~kb}$ )

Additional file 3: Questionnaire on menstrual patterns, fertility and reproductive outcomes after severe postpartum hemorrhage (control group). This file is the questionnaire that mailed to the patients with severe postpartum hemorrhage that were solely managed by uterotonic agents. (DOCX 29 kb)

Additional file 4: Dataset. This file is the dataset of this study. (XLSX 34 kb)

\section{Abbreviation}

$\mathrm{PPH}$ : Postpartum hemorrhage

\section{Acknowledgements \\ Not applicable.}

Funding

There is no funding for this study.

Availability of data and materials

The dataset used and/or analyzed during the current study are available as Additional file 4. 


\section{Authors' contributions}

KCW and TWWK have contributed to the intellectual planning of the project, carrying out of the experimental work, data analysis, writing of the manuscript and final approval of the contents. Both authors read and approved the final manuscript.

\section{Author's information}

Kong CW, MBChB, MRCOG, FHKAM. Consultant of Department of O\&G, United Christian Hospital, Hong Kong. Subspecialist in Maternal and Fetal Medicine.

Research interest on postpartum hemorrhage and balloon tamponade with a number of publications on this area.

To WWK, MBBS, MPH, M Phil, MD, FRCOG. Chief of Service, Department of O\&G, United Christian Hospital, Hong Kong. Subspecialist in Maternal and Fetal Medicine. Had more than 50 publications on research in obstetrics.

\section{Ethics approval and consent to participate}

Ethics approval for this study was granted by the Kowloon Central/ Kowloon East Ethics Committee Board of the Hospital Authority, Hong Kong (KC/ KE-16-0169/ER-1). Additional data was collected from the patients involved in the previous studies, for the current study. Consent was waived from the original studies, but written consent was obtained for the current study, and was attached to the questionnaire.

\section{Consent for publication}

Not applicable.

\section{Competing interests}

The authors declare that they have no competing interests.

\section{Publisher's Note}

Springer Nature remains neutral with regard to jurisdictional claims in published maps and institutional affiliations.

Received: 12 April 2018 Accepted: 8 November 2018

Published online: 21 November 2018

\section{References}

1. American College of Obstetricians and Gynecologists. ACOG practice bulletin: clinical management guidelines for obstetrician-gynecologists. Number 76, October 2006: postpartum hemorrhage. Obstet Gynecol. 2006; 108:1039-47.

2. Knight M, Callaghan WM, Berg C, Alexander S, Bouvier-Colle MH, Ford JB, et al. Trends in postpartum hemorrhage in high resource countries: a review and recommendations from the international postpartum hemorrhage collaborative group. BMC Pregnancy Childbirth. 2009:9:55.

3. Oyelese Y, Ananth CV. Postpartum haemorrhage: epidemiology, risk factors and causes. Clin Obstet Gynecol. 2010;53:147-56.

4. Al-Zirqi I, Vangen S, Forsen L, Stray-Pedersen B. Prevalence and risk factors of severe obstetric haemorrhage. BJOG. 2008:115:1265-72.

5. Condous GS, Arulkumaran S. Medical and conservative surgical management of postpartum hemorrhage. J Obstet Gynecol Can. 2003; 25:931-6.

6. Dabelea V, Schultze PM, RS MD Jr. Intrauterine balloon tamponade in the management of postpartum haemorrhage. Am J Perinatol. 2007;24:359-64.

7. Kong MC, To WW. Balloon tamponade for postpartum haemorrhage: case series and literature review. Hong Kong Med J. 2013:19:484-90.

8. Grönvall M, Tikkanen M, Tallberg E, Paavonen J, Stefanovic V. Use of Bakri balloon tamponade in the treatment of postpartum hemorrhage: a series of 50 cases from a tertiary teaching hospital. Acta Obstet Gynecol Scand. 2013; 92:433-8.

9. Varatharajan L, Chandraharan E, Sutton J, Lowe V, Arulkumaran S. Outcome of the management of massive postpartum hemorrhage using the algorithm "HEMOSTASIS". Int J Gynaecol Obstet. 2011;113:152-4.

10. Georgiou C. Menses, fertility and pregnancy following the use of balloon tamponade technology in the management of postpartum haemorrhage. Aust N Z J Obstet Gynaecol. 2014;54:287-90.

11. RCOG World congress 2017. https://epostersonline.com/rcog2017/node/ 1497. Accessed 19 Aug 2018.
12. Johanson R, Kumar M, Obhrai M, Young P. Management of massive postpartum haemorrhage: use of a hydrostatic balloon catheter to avoid laparotomy. Br J Obstet Gynaecol. 2001;108:420-2.

13. Nelson WL, O'Brien JM. The uterine sandwich for persistent uterine atony: combining the B-lynch compression suture and an intrauterine Bakri balloon. Am J Obstet Gynecol. 2007;196:e9-10.

14. Martin E, Legendre G, Bouet PE, Cheve MT, Multon O, Sentilhes L, et al. Maternal outcomes after uterine balloon tamponade for postpartum hemorrhage. Acta Obstet Gynecol Scand. 2015;94:399-404.

15. Kong CW, WWK T. Trends in conservative procedures and peripartum hysterectomy rates in severe postpartum haemorrhage. J Matern Fetal Neonatal Med. 2018;31:2820-6

16. Kong CW, WWK T. Prognostic factors for the use of intrauterine balloon tamponade in the management of severe postpartum hemorrhage. Int J Gynaecol Obstet. 2018:142:48-53.

17. World Health Organization. WHO recommendations for the prevention and treatment of postpartum haemorrhage. Geneva: World Health Organization; 2012. http://apps.who.int/iris/bitstream/handle/10665/ 75411/9789241548502_eng.pdf;jsessionid=2A87AFFC1B0E904D9232 F8B1721EAF92? sequence=1. Accessed 19 Aug 2018

18. B-Lynch C, Coker A, Lawal A, Abu J, Cowen MJ. The B-lynch surgical technique for the control of massive postpartum haemorrhage: an alternative to hysterectomy? Five cases reported. Br J Obstet Gynaecol. 1997:104:372-5.

19. Hayman R, Arulkumaran S, Steer PJ. Uterine compression sutures: surgical management of postpartum hemorrhage. Obstet Gynecol. 2002:99:502-6.

20. Doumouchtsis SK, Papageorghiou AT, Arulkumaran S. Systematic review of conservative management of postpartum hemorrhage: what to do when medical treatment fails. Obstet Gynecol Surv. 2007;62:540-7.

21. Gizzo S, Saccardi C, Patrelli TS, Di Gangi S, Breda E, Fagherazzi S, et al. Fertility rate and subsequent pregnancy outcomes after conservative surgical techniques in postpartum hemorrhage: 15 years of literature. Fertil Steril. 2013:99:2097-107.

22. Oberg AS, Hernandez-Diaz S, Palmsten K, Almqvist C, Bateman BT. Patterns of recurrence of postpartum hemorrhage in a large population-based cohort. Am J Obstet Gynecol. 2014;210(229):e1-8.

23. Poggi $\mathrm{SH}$, Yaeger A, Wahdan $\mathrm{Y}$, Ghidini A. Outcome of pregnancies after pelvic artery embolization for postpartum hemorrhage: retrospective cohort study. Am J Obstet Gynecol. 2015;213:576.e1-5.

24. Nizard J, Barrinque L, Frydman R, Fernandez H. Fertility and pregnancy outcomes following hypogastric artery ligation for severe post-partum haemorrhage. Hum Reprod. 2003;18:844-8.

25. Hardeman S, Decroisette E, Marin B, Vincelot A, Aubard Y, Pouquet M, et al. Fertility after embolization of the uterine arteries to treat obstetrical hemorrhage: a review of 53 cases. Fertil Steril. 2010;94:2574-9.

26. Poujade O, Grossetti A, Mougel L, Ceccaldi PF, Ducarme G, Luton D. Risk of synechiae following uterine compression sutures in the management of major postpartum haemorrhage. BJOG. 2011:118:433-9.

27. 33rd Annual Meeting of the Society for Maternal-Fetal Medicine: The Pregnancy Meeting. https://www.ajog.org/article/S0002-9378(12)01806-6/ fulltext. Accessed 19 Aug 2018.

Ready to submit your research? Choose BMC and benefit from:

- fast, convenient online submission

- thorough peer review by experienced researchers in your field

- rapid publication on acceptance

- support for research data, including large and complex data types

- gold Open Access which fosters wider collaboration and increased citations

- maximum visibility for your research: over $100 \mathrm{M}$ website views per year

At $\mathrm{BMC}$, research is always in progress.

Learn more biomedcentral.com/submissions 